\title{
IMPLEMENTAÇÃo E ANÁLISE DE UM ALGORITMO DE CONTROLE TOLERANTE A FALHAS COM CONSIDERAÇÕES SOBRE RESTRIÇÕES OPERACIONAIS
}

\author{
K. L. RESENDE ${ }^{1}$ e T. V. DA COSTA ${ }^{1}$ \\ ${ }^{1}$ Universidade Federal de Itajubá, Instituto de Recursos Naturais - Engenharia Química \\ E-mail para contato: karinelresende@gmail.com
}

\begin{abstract}
RESUMO - As indústrias químicas estão sujeitas à ocorrência de falhas que afetam seu desempenho e segurança. O sistema de controle da planta possui malhas integradas e uma falha pode encadear prejuízos a todo o processo. Assim, as indústrias têm se dedicado a implementar controladores tolerantes para manter um desempenho aceitável em cenários de falha. Neste contexto, o objetivo deste trabalho foi aplicar a teoria dos atuadores virtuais considerando restrições operacionais aplicadas no desvio entre as saídas do processo em falha e seu comportamento nominal. O algoritmo proposto foi aplicado a um reator contínuo agitado (CSTR) sujeito a falha na malha de controle. Os resultados indicaram que, mesmo sob falha, o algoritmo desenvolvido mantém o desempenho do processo aceitável, dentro dos limites definidos para o desvio em relação a uma referência.
\end{abstract}

\section{INTRODUÇÃO}

As plantas e equipamentos inerentemente seguros são projetados para evitar ou conter condições anormais de operação, minimizando a ocorrência de acidentes. Neste contexto, as indústrias estão investindo cada vez mais em técnicas capazes de impedir que a propagação de uma falha afete a segurança e o desempenho do processo.

O controle da produção é realizado por sistemas de controle com malhas integradas e acopladas e uma única falha pode prejudicar todo o processo. Deste modo, uma das formas para garantir o desempenho aceitável do processo mesmo em cenário de falhas é introduzir aspectos de segurança aos sistemas de controle, tornando-os uma das camadas de proteção do processo.

O controle tolerante a falha é uma classe de controlador cuja função é conter o avanço da falha e manter o desempenho do processo satisfatório e estável. Este controlador ainda não é muito utilizado por precisar de algoritmos complexos e de instrumentação e processadores de alto desempenho (ZUBEN, 2003). Entretanto, ele vem ganhando espaço na aplicação fabril.

Dentre as estratégias de controle tolerante está o atuador virtual, utilizado quando existe mal funcionamento ou perda de atuadores. Este método fundamenta-se na redistribuição dos sinais de controle para os atuadores ainda disponíveis e no ocultamento da falha do controlador nominal através do desvio entre as saídas reais e estimadas da planta (BLANKE, 2006).

Desse modo, o objetivo desse trabalho foi desenvolver no software Scilab um algoritmo de controle tolerante adotando a estratégia de atuador virtual na forma de um problema de 
otimização, com foco na aplicação de restrições operacionais (rígida e com folga) no desvio entre o processo em falha e nominal. Para validação, implementou-se o código no sistema de controle multivariável de um reator CSTR com falha em uma de suas malhas de controle.

\section{METODOLOGIA}

\subsection{Fundamentação Teórica}

O método de reconfiguração baseado em atuadores virtuais, ilustrado na Figura 1, oculta a falha do controlador nominal através do sinal de saída $y_{m}$, reconstruído a partir do sinal original $y_{p}$. Para isso, um bloco reconfigurador é inserido entre o controlador nominal e o processo. Esse bloco compara as saídas reais com as teóricas e calcula o desvio entre elas.

Figura 1 - Esquema do controle tolerante com bloco reconfigurador

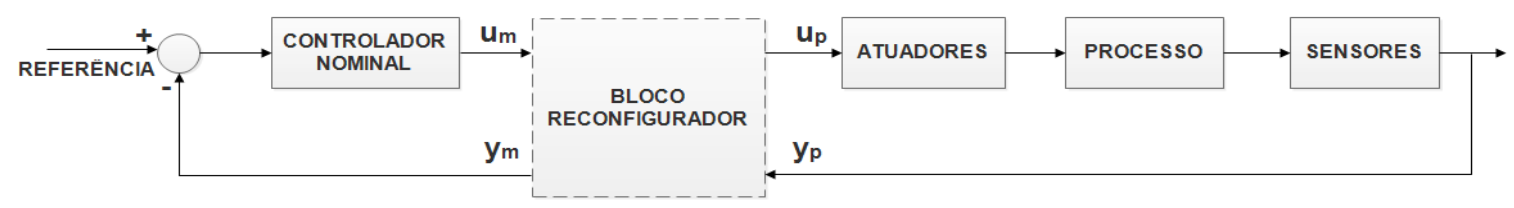

A interpretação da técnica dos atuadores virtuais com horizonte móvel (MHVA, Moving Horizon Virtual Actuators) permite reescrever a equação original proposta por Steffen (2005) como uma otimização (Equação 1), que minimiza o desvio da planta em falha de sua operação normal a partir do vetor $x_{\Delta}$, ponderado pela matriz $Q_{F}$, somado ao esforço de controle dos atuadores remanescentes, ponderados pela matriz $R_{F}$ (Costa et al, 2013).

$$
\begin{gathered}
\min _{u_{p}} J=\sum_{i=1}^{N} \hat{x}_{\Delta}^{T}(k+i \mid k) Q_{F} \hat{x}_{\Delta}(k+i \mid k)+u_{p}^{T}(k+i-1 \mid k) R_{F} u_{p}^{T}(k+i-1 \mid k) \\
\text { sujeito à: } \quad \begin{array}{c}
\hat{x}_{m}(k+i \mid k)=A_{m} \hat{x}_{m}(k+i-1 \mid k)+B_{m} u_{m}(k+i-1 \mid k) \\
\hat{x}_{p}(k+i \mid k)=A_{p} \hat{x}_{p}(k+i-1 \mid k)+B_{p} u_{p}(k+i-1 \mid k) \\
\hat{x}_{\Delta}(k+i \mid k)=\hat{x}_{m}(k+i \mid k)-\hat{x}_{p}(k+i \mid k) \\
\hat{x}_{\Delta}(k \mid k)=0 \\
u_{\min } \leq u_{p}(k+i-1 \mid k) \leq u_{\max }
\end{array}
\end{gathered}
$$

No problema de otimização, $x_{m}$ e $u_{m}$ são os estados e as entradas do modelo nominal (livre de falhas), $x_{p}$ e $u_{p}$ os estados e entradas da planta em situação de falha e $x_{\Delta}$ o desvio com relação ao comportamento nominal. As matrizes $A_{m}, A_{p}, B_{m}$ e $B_{p}$ são as matrizes dos estados e saídas dos respectivos modelos em espaço de estados. O problema é sujeito às restrições de igualdade que incluem os modelos citados, ao desvio $x_{\Delta}$ considerado nulo (em variável desvio) para situações normais de operação e às faixas dos atuadores remanescentes (limites $u_{\max } \mathrm{e} u_{\text {min }}$ ).

Para a reconstrução do sinal, considera-se as equações de saída do espaço de estados para os modelos com e sem falha, onde se soma o desvio $x_{\Delta}$ no sinal de saída do processo, ocultando a falha do controlador nominal, conforme a Equação 2.

$$
y_{m}(k)=y_{p}(k)+C x_{\Delta}(k)
$$

Neste trabalho, além das restrições impostas nas variáveis manipuladas, impedindo a sobrecarga dos atuadores remanescentes, propôs-se o uso de restrições no vetor $x_{\Delta}$ como forma 
de impor limites considerados seguros para o desvio da planta com relação à sua operação normal. Assim, restrições do tipo $x_{\Delta} \in \mathbb{X}$ (Equação 3) foram acrescentadas ao atuador virtual.

$$
\mathbb{X}:=\left\{x_{\Delta}(k+i \mid k) \mid x_{\Delta, \min } \leq x_{\Delta}(k+i-1 \mid k) \leq x_{\Delta, \max }\right\}
$$

O problema apresentado em Costa et al. (2013) foi acrescido de limites na variável $x_{\Delta}$ e as restrições (Equação 3) de variáveis folga. A consideração da não factibilidade da solução consiste na adição de um termo de penalização na Equação 1, resultando nas Equações 4 e 5.

$$
\begin{gathered}
J=\sum_{i=1}^{N} \hat{x}_{\Delta}^{T}(k+i \mid k) Q_{F} \hat{x}_{\Delta}(k+i \mid k)+u_{p}^{T} R_{F} u_{p}^{T}+\epsilon^{T} W \epsilon \\
x_{\Delta, \min }-\epsilon \leq \hat{x}_{\Delta}(k+i-1 \mid k) \leq x_{\Delta, \max }+\epsilon \\
\epsilon \geq 0
\end{gathered}
$$

\subsection{Aplicação em um Reator Contínuo Agitado}

O exemplo ilustrado na Figura 2 (HOVD E SKOGESTAD, 1994), foi selecionado para análise do atuador virtual projetado e considera restrições nos atuadores remanescentes e limites no desvio das variáveis de saída em relação ao desempenho nominal. O processo consiste em um reator contínuo agitado (CSTR) encamisado, onde ocorre a reação exotérmica $\mathrm{A} \longrightarrow \mathrm{B}$.

Figura 2 - Diagrama de instrumentação do CSTR com camisa de resfriamento

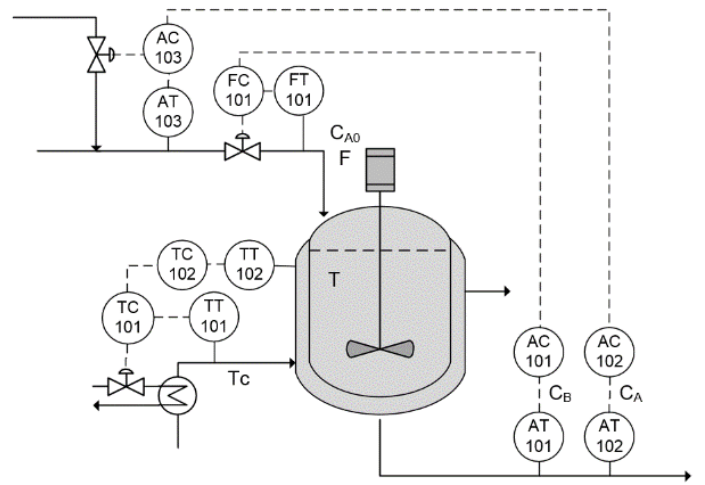

O objetivo do controlador nominal é manter a concentração do reagente $\mathrm{C}_{\mathrm{A}}\left(\mathrm{kmol} / \mathrm{m}^{3}\right)$, do produto $\mathrm{C}_{\mathrm{B}}\left(\mathrm{kmol} / \mathrm{m}^{3}\right)$ e a temperatura do reator $\mathrm{T}(\mathrm{K})$ em suas referências, manipulando a vazão de alimentação $F\left(\mathrm{~m}^{3} / \mathrm{min}\right)$, a concentração inicial de reagente $\mathrm{C}_{\mathrm{A} 0}\left(\mathrm{kmol} / \mathrm{m}^{3}\right)$ e a temperatura da água de resfriamento da camisa $\mathrm{T}_{\mathrm{c}}(\mathrm{K})$. $\mathrm{O}$ pareamento sugerido foi: Malha 1 $\left(\mathrm{C}_{\mathrm{A}}-\mathrm{C}_{\mathrm{A} 0}\right)$, Malha $2\left(\mathrm{C}_{\mathrm{B}}-\mathrm{F}\right)$ e Malha $3\left(\mathrm{~T}-\mathrm{T}_{\mathrm{c}}\right)$. Adotou-se controladores nominais do tipo PID, com os parâmetros $K_{c 1}=10 ; \tau_{i 1}=10 ; \tau_{d 1}=0$ para a primeira malha, $K_{c 2}=-10 ; \tau_{i 2}=$ $10 ; \tau_{d 2}=0$ para a segunda malha e $K_{c 3}=40 ; \tau_{i 3}=10 ; \tau_{d 3}=0$ para a terceira malha.

Além disso, aplicou-se restrições nas variáveis de entrada: $-2,619 \mathrm{kmol} / \mathrm{m}^{3} \leq u_{1} \leq 15$ $\mathrm{kmol} / \mathrm{m}^{3},-3 \mathrm{~m}^{3} / \mathrm{min} \leq u_{2} \leq 1 \mathrm{~m}^{3} / \mathrm{min}$ e $-12 \mathrm{~K} \leq u_{3} \leq 6,4 \mathrm{~K}$ e incorporou-se ao problema de otimização restrições no desvio da planta em relação ao seu estado nominal, o que pode representar limites de segurança para essas variáveis em um sistema real. Isto implica em acrescentar restrições no estado $x_{\Delta}$ ao problema de programação quadrática $(\mathrm{QP}$, Quadratic Programming) originalmente concebido para o MHVA. As restrições adotadas foram: -1 $\mathrm{kmol} / \mathrm{m}^{3} \leq x_{\Delta 1} \leq 0,2 \mathrm{kmol} / \mathrm{m}^{3},-0,01 \mathrm{kmol} / \mathrm{m}^{3} \leq x_{\Delta 2} \leq 0,02 \mathrm{kmol} / \mathrm{m}^{3} \mathrm{e}-2 \mathrm{~K} \leq x_{\Delta 3} \leq 1 \mathrm{~K}$. 


\section{RESULTADOS E DISCUSSÃO}

\subsection{Desempenho do Processo Nominal e em Cenário de Falha}

A falha adotada neste estudo consistiu na perda total do atuador de vazão da malha de controle 2 com bloqueio no estado estacionário. Neste caso, o atuador virtual tem então apenas dois atuadores disponíveis (referentes à $C_{A_{0}}$ e $T_{c}$ ) para manter $C_{B}$ na referência.

A Figura 3 ilustra em (a), (b), (c) as variáveis controladas e em (d), (e), (f) as manipuladas, para o controlador nominal PID em condições de operação normal (curva preta) e em cenário de falha (curva vermelha). A simulação considerou um tempo de amostragem de $\mathrm{T}_{\mathrm{s}}=0,1 \mathrm{~min} \mathrm{e}$ partiu de condições iniciais iguais ao estado estacionário do processo.

Figura 3 - Comportamento do processo com controlador nominal PID
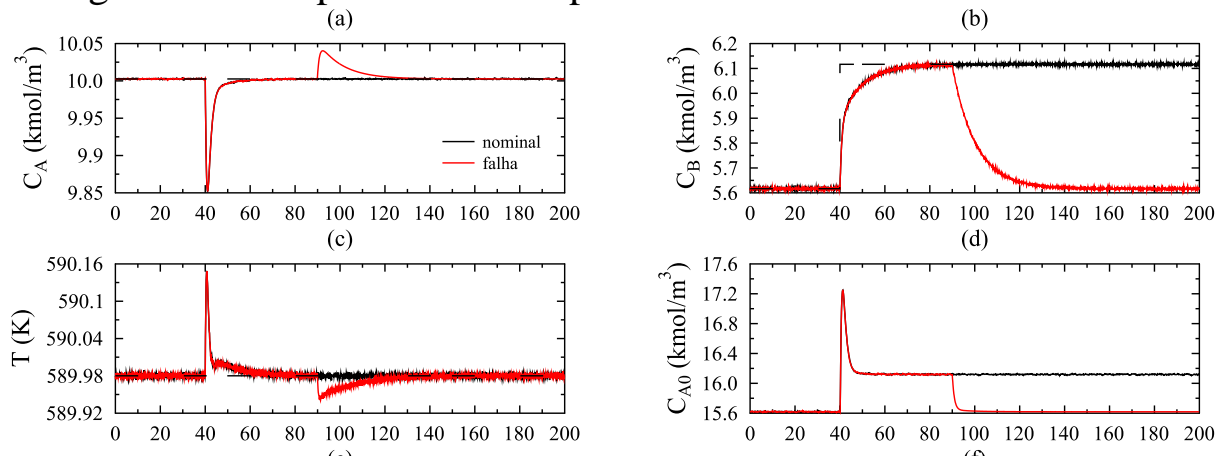

(e)
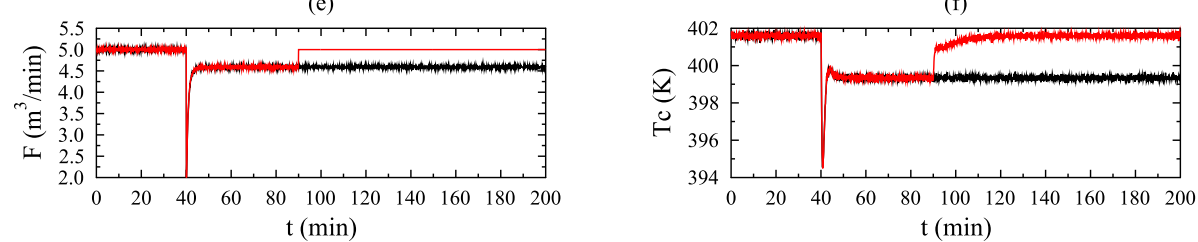

Constata-se das figuras $3 \mathrm{a}$, $3 \mathrm{~b}$ e $3 \mathrm{c}$ que os controladores PID estavam bem sintonizados, pois as variáveis de saída $\left(C_{A}, C_{B}\right.$ e $\left.T\right)$ atingiram suas referências. A interação entre as malhas de controle é percebida devido à alteração na referência de $C_{B}$ ter afetado $C_{A}$ e $T$. Apesar disso, foi possível manter $C_{A}$ e $T$ em suas referências, pois os controladores estão bem sintonizados.

Como as malhas de controle são acopladas, a falha afetou todas as variáveis, sendo perceptível o momento de sua ocorrência ( $\mathrm{t}=90 \mathrm{~min}$ ) nas figuras 3 (b), (d), (e) e (f). Os PID's conseguiram manter $C_{A}$ e $T$ em suas referências, mas $C_{B}$ se afastou do valor desejado. Logo, o controlador PID não conseguiu manter um desempenho satisfatório após a ocorrência da falha.

\subsection{Implementação do Atuador Virtual com Restrições em $x_{\Delta}$}

Para manter a concentração $C_{B}$ na referência mesmo no cenário de falha, implementouse o atuador virtual com restrições em $x_{\Delta}$. A restrição na variável desvio $\left(x_{\Delta}\right)$ define o quanto a variável controlada pode se afastar do seu desempenho nominal. Mesmo com um menor número de atuadores disponíveis, as saídas do sistema devem respeitar a faixa de operação e os limites considerados seguros para o processo. Na Figura 4, (a), (b), (c), (g), (h) e (i) ilustram o processo reconfigurado a partir do atuador virtual e (d), (e) e (f), a aplicação da restrição rígida em $x_{\Delta}$. 
Figura 4 - Processo com atuador virtual e restrição rígida
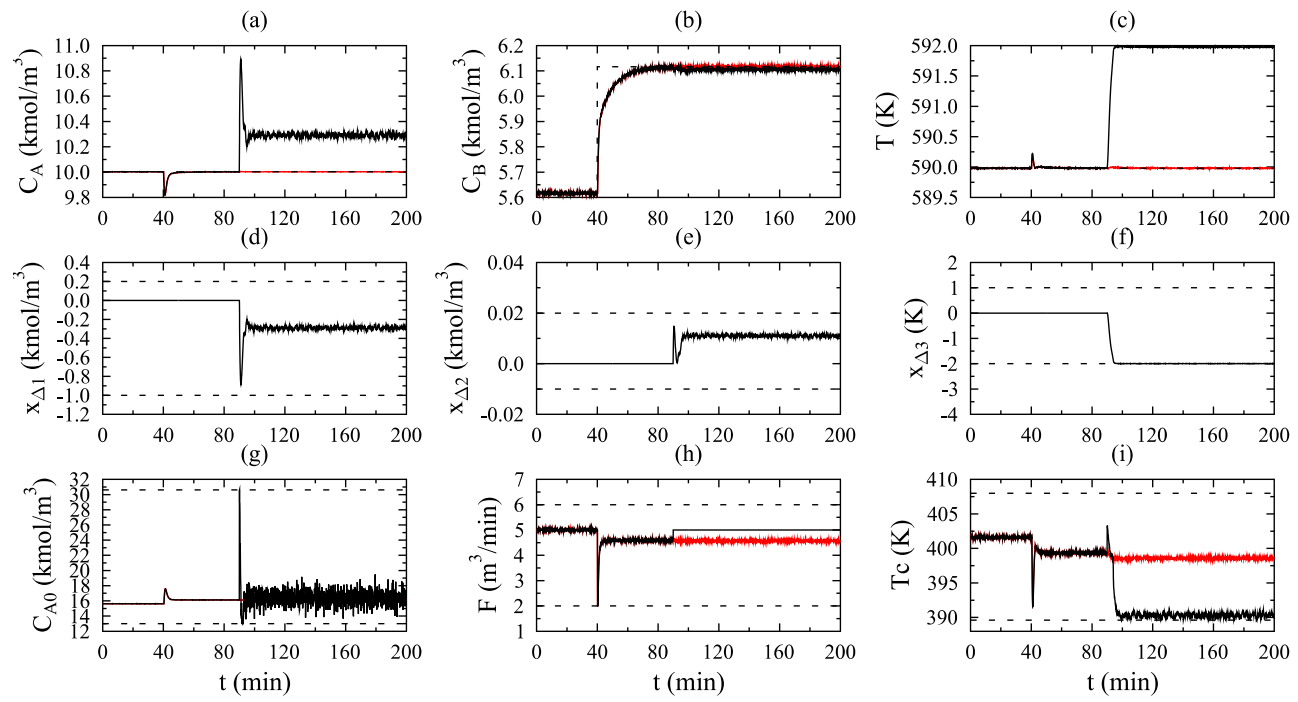

Nas figuras 4 (g), (h) e (i) a curva preta representa o valor real das variáveis manipuladas e a curva vermelha, o sinal reconstruído pelo atuador virtual para ocultar a falha do controlador nominal. $\mathrm{O}$ atuador virtual manteve a variável $\mathrm{C}_{B}$ no valor esperado (figura $4 \mathrm{~b}$ ) mesmo sob falha. A esta variável $\left(C_{B}\right)$ foi atribuída maior importância, afastando os demais estados $\left(C_{A}\right.$ e T) de suas referências (figuras 4 a e c). Os atuadores remanescentes se dedicaram então a manter $\mathrm{C}_{\mathrm{B}}$ na referência, deixando as demais variáveis controladas em segundo plano.

Para controlar $\mathrm{C}_{\mathrm{B}}$ com a variável de entrada da malha $3\left(\mathrm{~T}_{\mathrm{c}}\right)$, a temperatura teria que ultrapassar o limite imposto. Como a restrição é rígida, isso não é possível (figura $4 \mathrm{f}$ ) e agora resta apenas o atuador de $\mathrm{C}_{\mathrm{Ao}}$ para controlar $\mathrm{C}_{\mathrm{B}}$. Esse tipo de restrição pode tornar o problema de otimização infactível, pois pode não existir valor possível que não viole a restrição imposta.

Analogamente, a figura 5 (d), (e), (f) ilustra a aplicação da restrição com folga em $x_{\Delta} \mathrm{e}$ 5 (a), (b), (c), (g), (h), (i), o processo com a implementação do atuador virtual.

Figura 5 - Processo com atuador virtual e restrição com folga
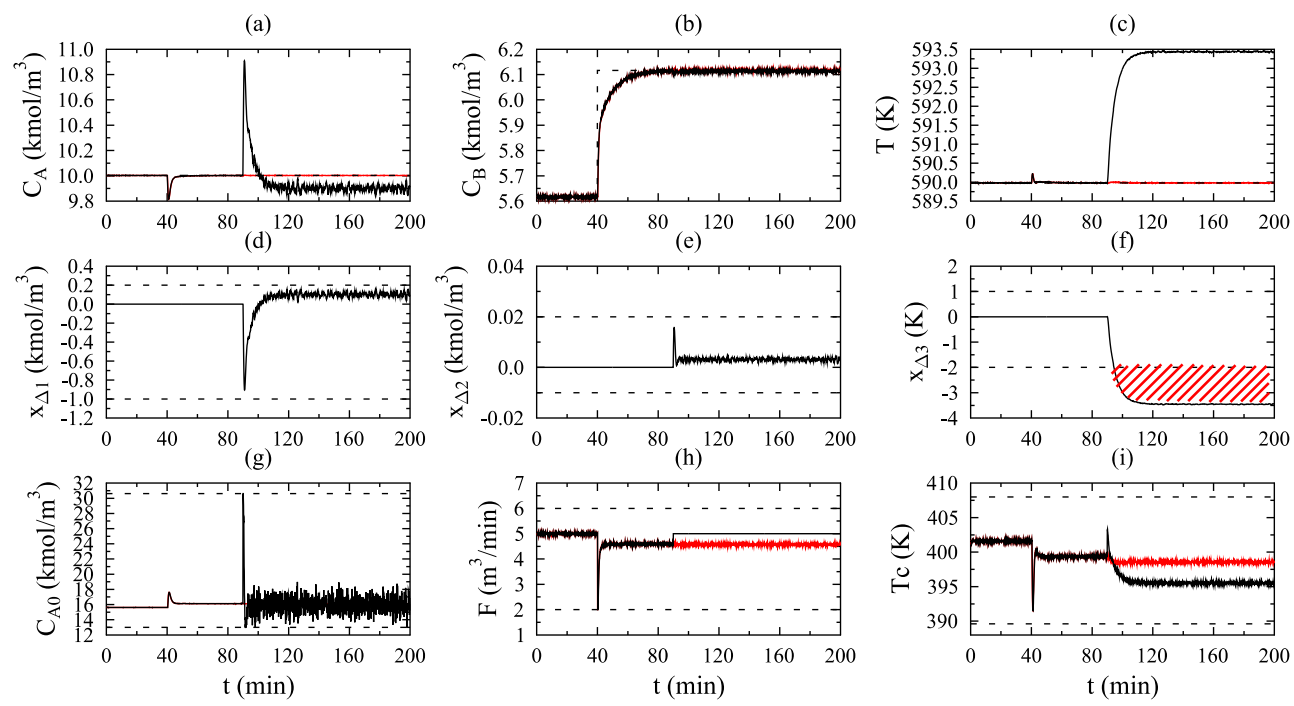
Nota-se que a variável folga permite que a variável controlada $(T)$ ultrapasse a restrição quando necessário (área hachurada na figura 5f), penalizando a função objetivo do projeto do controlador tolerante. Isto evita a não factibilidade da otimização, pois a folga permite a ultrapassagem do limite estabelecido, garantindo que uma nova solução seja calculada.

Portanto, o controlador tolerante desenvolvido foi eficiente, pois manteve o desempenho satisfatório na presença de falha. A implementação de restrição nos desvios em relação ao comportamento nominal permitiu simular restrições operacionais reais, sendo que a restrição com folga evita a infactibilidade do problema de otimização do atuador virtual.

\section{CONCLUSÃO}

Este trabalho consistiu no desenvolvimento e avaliação de um algoritmo de controle tolerante baseado na estratégia de atuador virtual com horizonte móvel, considerando a imposição de restrições no desvio entre a planta com falha e seu comportamento nominal. $\mathrm{O}$ algoritmo desenvolvido ocultou a falha do controlador nominal e manteve as variáveis próximas às referências mesmo com a perda de um atuador. Os resultados demonstram que a abordagem é viável e permite que restrições operacionais sejam acrescentadas à reconfiguração do controlador. As restrições rígidas podem deixar o problema de otimização aqui apresentado sem solução, ao passo que as restrições com folga garantem sua factibilidade.

A escolha do tipo (rígida ou não rígida) e das faixas das restrições deve ser feita segundo a análise das variáveis controladas, observando os aspectos econômicos e principalmente a segurança do processo. As variáveis críticas com relação a esses critérios devem ser priorizadas para que permaneçam em suas referências ou em faixas pré-determinadas sem o uso de variáveis folga. Para as variáveis não críticas (permitem perda de desempenho) pode-se utilizar restrições não rígidas, resultando em maior flexibilidade em caso de falhas. Neste sentido, os trabalhos futuros contemplam, com base em critérios de segurança, desempenho e operabilidade, estabelecer prioridades para as variáveis do processo, especificar quais devem ser mantidas em suas referências e a faixa do desvio permitido para as demais variáveis.

\section{REFERÊNCIAS}

BLANKE, M., KINNAERT, M., LUNZE, J., STAROSWIECKI, M. \& SCHRÖDER, J. Diagnosis and Fault-Tolerante Control. Springer. Berlin, 2006.

COSTA, T.; FILETI, A.; SILVA F.; OLIVEIRA-LOPES, L. Control Reconfiguration of Chemical Processes Subjected to Actuator Faults: A Moving Horizon Approach. In: Intelligent Systems and Control. Marina del Rey. Calgary: Actapress. 2013.

HOVD, M.; SKOGESTAD, S. Pairing Criteria for Decentralized Control of Unstable Plants. Chemical Engineering University of Trondheim (NTH). Trondheim, 1994.

STEFFEN, T. Control Reconfiguration of Dynamical Systems: Linear Approaches and Structural Tests. Springer. Heidelberg, 2005.

ZUBEN, J. V. Tópicos Avançados: Controle Robusto, Controle Adaptativo e Controle Inteligente. DCA, FEEC - Universidade Estadual de Campinas. Campinas, 2003. 